\title{
Climate and Vine - Representation and communication of spatio-temporal relationships in the web application AustrianVineyards.com
}

\author{
Dominik Wieser $^{\mathrm{a}, *}$, Karel Kriz ${ }^{\mathrm{b}}$ \\ ${ }^{a}$ Department of Geography and Regional Research, University of Vienna, dominik.wieser@univie.ac.at \\ ${ }^{b}$ Department of Geography and Regional Research, University of Vienna, karel.kriz@univie.ac.at \\ * Corresponding author
}

\begin{abstract}
A central component of the web-based map application AustrianVineyards.com is the communication of the climatic conditions prevailing in the individual wine-growing regions. To accomplish this, it is necessary to obtain the relevant data and process it so that it can be converted into high-quality visualisations. Therefore, the goal is to develop a climate communication tool that is as flexible as possible, so that it is possible to display several meteorological parameters. Moreover, this visualisation should be appealing to experts as well as to laypersons. This paper shows how the data preparation and visualisation is managed so that these demands can be met. The result of the workflow are versatile climate and weather charts that are easy to interpret thanks to the inclusion of historical data. Furthermore, geocommunicative aspects are a central part of the concept through their assignment to wine origins from AustrianVineyards.com and visual properties that make the diagrams comparable with one another.
\end{abstract}

Keywords: climate, geo-communication, thematic maps, wine

\section{Introduction}

AustrianVineyards.com is a map-based communication portal for the origin of Austrian wines (Figure 1; Kriz and Pucher 2021; AustrianVineyards.com). Numerous morphological parameters are displayed for individual winegrowing regions. Together with other factors such as climate, they determine the terroir of a wine. Therefore, the effective communication of climate-related data is an important topic that is being pursued in connection with the development of the application. This raises the question of the optimal visualization of climatological characteristics, so that wines with their different sites can be best attributed to the climatic conditions to which they are exposed. Since the vintages of the wines are important, due to the climatic characteristics that vary from year to year, the temporal aspects should be considered in addition to the spatial ones. Consequently, the design of the presentation methods should be such that both spatial and temporal visual comparability is provided.

Against this background, the current discourse on methods for adequate spatio-temporal climate communication will be presented. For this purpose, the associated problem areas are first outlined. Subsequently, the characteristics of climate diagrams as a specific climate communication method are discussed by focusing on individual elements such as colour schemes, the possible inclusion of icons and interactivity.

Furthermore, existing web applications for climate geocommunication are briefly introduced to present existing approaches for visual climate-geo-communication. Based on these insights, AustrianVineyards.com is designed to communicate the climate with emphatic consideration of spatial aspects.

In this respect, the methodical implementation is presented considering the requirement to use exclusively open data, especially since there are specific problem areas in the data acquisition and handling. Regarding the actual visualizations, reference is made to the demand of the "image of excellence," which AustrianVineyards.com is supposed to serve as a wine marketing platform. Consequently, aesthetic aspects play an essential role.

The results are two types of diagrams for the visualization of different meteorological parameters, which can be accessed via the web map of AustrianVineyards.com to ensure the geo-communication. After briefly introducing them and their characteristics, the article concludes with a discussion recalling the state of research. Thereby, the innovative features of the climate charts in AustrianVineyards.com should become visible.

\section{Spatio-temporal climate communication}

Data visualization is considered a "key technology" for communicating and understanding climatic issues. Therefore, the number of visual implementations for this very purpose is extensive. Particularly in the context of climate change and the monitoring of the climatic situation on earth, the use of graphics to communicate this information is common practice, especially since the human perceptual system is particularly capable of recognizing changes in patterns and can therefore quickly perceive shapes. Climate graphics are considered 


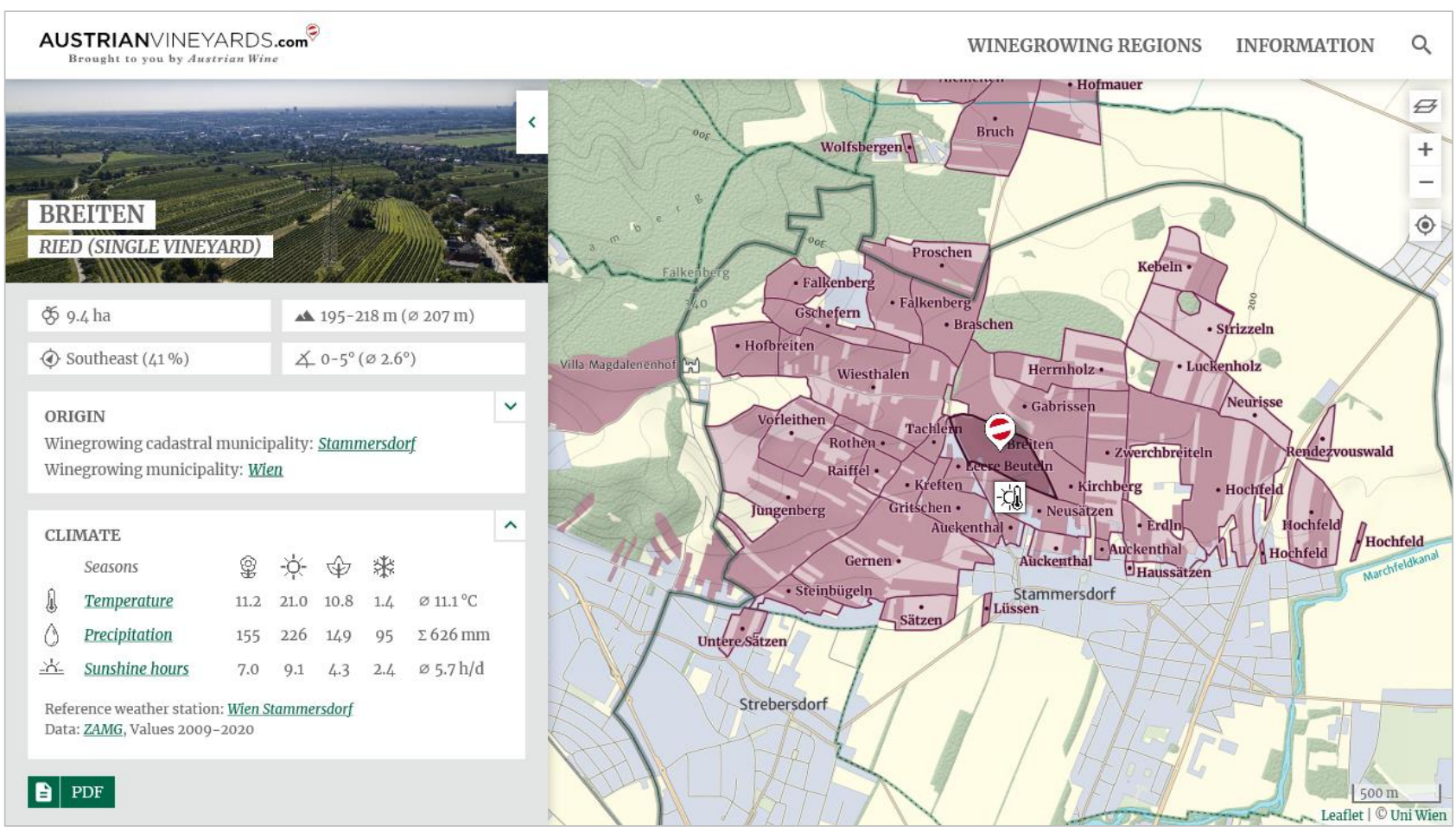

Figure 1. Screenshot of the AustrianVineyards.com web app. Climate data is displayed in the info box. If they are clicked, climate diagrams are shown. In the map, an icon shows the location of the corresponding climate station.

successful if they present complex concepts as directly as possible, in an informative, aesthetically appealing, and comprehensible way, so that the knowledge they contain can be conveyed to anybody in an "easy to digest" manner (Schneider 2012).

\subsection{Challenges in climate communication}

A special quality of climate communication lies in the fact that issues are presented which can only be perceived indirectly. Long-term developments such as changes in average temperature over several decades are beyond the scope of human experience. Information graphics, however, open the opportunity to make such things comprehensible by making genuinely invisible phenomena literally visible (Schneider 2012). In this sense, appropriate visualizations can establish "a direct interface between digital data in a computer and the human perceptual and cognitive abilities" (Nocke 2014) in a way that presents abstract relationships compactly and intuitively.

A major problem in visualization in general and of climate data in particular is that the representation of the data is necessarily always subject to limitations and thus cannot reflect all facets. As a result, aspects such as statistical aggregation of the data remain mostly hidden. In this context, Nocke (2014) uses the figure of speech of the iceberg, of which only the tip is visible: climate visualizations are always the compressed result of the processing of data that have already undergone numerous compression steps beforehand. Nevertheless, interactive visualizations can expand the amount of data shown or accessible in comparison to static visualizations, for example, by enabling users to have additional temporal information displayed (Wrobel and Reusser 2014).

Furthermore, when representing climatic situations, it should be noted that climate data are genuinely spatial data, since they always have their validity (during a specific point in time or space) for a specific geographical point or area. Therefore, a geo-communicative momentum is inherent to every form of climate communication. Accordingly, the underlying information is "geo-spatial data" (Eichner et al. 2015). For that reason, geographic maps are also suitable for displaying such data. Climatic characteristics can then be directly assigned to a point or area on a map. Thus, while diagrams are commonly used to express the quantitative manifestations of climate data, maps are used to focus on the spatial aspects (Zeptner et al. 2021). By combining both approaches, the climate-geocommunication is complete.

However, and especially regarding computer-generated infographics, the visual presentation forms should not necessarily be a continuation of established implementation examples, as these often lack a cognitivepsychological design foundation (Harold et al. 2016). Nevertheless, to achieve results that meet the requirements of the human visual perception system, Harold et al. (2016) compiled based on psychological findings "Evidenceinformed guidelines to improve accessibility of scientific graphics of climate science:" Essentially, only the information needed for the communication intent should be displayed. Important graphical elements should be highlighted to make them the main focus of attention. Sensibly used text modules help with the interpretation of the graphics. In the case of interactivity, recipients should 
be given control over when and which additional components are shown. Visual representations should be consistent with metaphors that support conceptual thinking. Text that is directly related to graphic elements should be placed appropriately close to them. In addition, text can help recipients to understand diagrams by providing interpretive guidance.

\section{2 (Possible) components of climate charts}

A typical field of application for diagrams is the presentation of climate data. According to Allaby (2019), these are "two-dimensional, graphical representation[s] of one major climatic element against another," so that a "quick visual comparison of the climates of different areas" is possible. This also brings the importance of the spatial reference to the force. Climate data in the sense of data depicted in climate diagrams are generally "data based on measurements and observations in the past and their statistical parameters, which are used to describe the climate" (Deutscher Wetterdienst). The visual communication of climate-related parameters and their geographical manifestations has a tradition dating back to the $19^{\text {th }}$ century (Kaye et al. 2012).

\subsubsection{Colours}

In data visualisation, colours are particularly important as "visual attributes" of charts (Harper and Agrawala 2017), as they are the most powerful of all visual variables (Moody 2009). If used correctly, they can help to facilitate the analysis of the information presented, such as meteorological data. Conversely, even the communication of simple facts risks failure if the choice of colour is misleading (Stone 2016).

While the sum of colours perceivable by humans is summarised as absolute colour spaces, non-absolute colour spaces are self-contained colour models such as RGB, CMYK or HSL/HSV, which are primarily related to input and output devices such as cameras or screens (Zeller and Rogers 2020); accordingly, they are used in digital diagram drawing.

When compiling adequate colour palettes, in addition to the properties of the data (e.g. scale level), attention should also be paid to finding a harmonious colour combination, for which different tools can be used (Stone 2016; Zeileis and Hornik 2006).

If colours are combined in a rule-guided way, this facilitates a faster and less concentration-requiring reception for the recipient. Furthermore, there is empirical evidence that colours that can be named are easier to remember. In this sense, an appropriate choice of colours enables maps and diagrams to be generated that are intuitively easier to read (Kim et al. 2020; Samsel et al. 2019).

\subsubsection{Icons}

In addition to the targeted use of selected colour palettes, icons can help to support the interpretation of (climate) diagrams. Icons are visual representations of any object or idea. Their primary purpose is to communicate meaning within a graphical user interface. There are numerous reasons for using icons, as they take up little space but can still be perceived quickly under certain conditions. In addition, they are language-independent, which eliminates the need for translation in multilingual applications. Furthermore, they are easy to click, which is particularly important when interacting via touchscreen. Finally, the use of icons creates the fundamental potential that they can be designed in a visually appealing way (Harley 2014). The latter can commonly be achieved using certain visual principles. These include balance, contrast, and appropriate line management (Kimball 2013; Kascak et al. 2013).

A common distinction can be made between symbolic and iconic icons. Symbolic icons offer the advantage that they speed up the recognition process and are more likely to be correctly understood even by a non-expert audience. Finally, their importance should not be underestimated in favour of aesthetic aspects, as they can be decorative in addition to their communicative function. While icons are used almost ubiquitously as signatures in cartography, which is also due to a tradition of know-how building within the discipline, their potential is often not exploited by software-supported diagrammatic representations (Moody 2009), which is why Moody (2009) concludes: "Cartographers are masters of graphical representation and notation designers can learn much from them."

However, only a few icons are considered "universally" understandable, as there is usually no uniform standard. Rather, numerous pictograms are often ambiguous. Even icons that are particularly widespread are not always used for the same purpose. An example is the hamburger icon, which is usually used to access the main menu; occasionally it is used (in a very similar form) to symbolise lists (Harley 2014). To address this issue, Harley (2014) insists that icons should always be labelled to minimise misunderstandings. The labels should always be visible, even without interaction with the users.

\subsubsection{Interactivity}

Static visualisations can only illustrate an unchanging representation version of the data to be communicated. This limits, for example, the number of dimensions that can be represented. Under certain circumstances, this makes it necessary to string several static images together to evoke a certain intended narrative of the information depicted or to take all desired dimensions into account. Accordingly, the representation of multidimensional data sets in static visualisations proves to be particularly challenging. However, the use of static diagram images remains unquestioned for certain purposes, such as the illustration of less complex data or as an image in print media. For interactive approaches, though, it turns out to be useful during numerous use cases in which, for example, users are to be enabled to explore the data independently (Murray 2017).

A major advantage of interactive graphics is that they are suitable both for communicating an overview of a subject and for conveying more detailed information about the same subject, because they offer users who are interested in more in-depth content the option of actively retrieving 
more in-depth units of knowledge through interaction. For this reason, interactive visualisations can address a broader audience than it would be the case with just static illustrations. Last but not least, interactive diagrams are able to encourage the recipients to engage further with the data, whereas non-interactive graphics lack this potential (Murray 2017). All in all, these properties of interactive data visualisations mean that the understanding of the data can be increased. This is equally true for information graphics in a scientific context as well as for graphics that are intended to reach a broad audience (Wrobel and Reusser 2014).

The concrete design possibilities of interactive elements in data visualisations are manifold. Grant (2019) mentions in this context, among other things, the display of tooltips when hovering the mouse pointer over certain elements, clicking to display further information, zooming by clicking or - in the case of touch operation-'pincer movements' with the fingers, scrolling, switching ("toggle") certain information on and off, clicking through successive units of a "story" by means of arrow buttons provided for this purpose or-in the case of mapschanging the scale.

\subsection{Existing Applications}

Numerous online platforms exist (or have existed) for the communication of climate data. Examples include the Earth Observation Monitor (till 2020; Earth Observation Monitor; Eberle 2016), the Global Climate Monitor (Camarillo-Naranjo et al. 2019; Global Climate Monitor), OpenSensorWeb (OpenSensorWeb) or ClimateCharts.net (ClimateCharts.net; Wiemann et al. 2016; Zeptner et al. 2021). Although climate is treated as an exclusive topic on these websites, whereas on AustrianVineyards.com climate represents one of several topic complexes, the basic problem of implementing climate geocommunication using diagrammatic representations is comparable to that of AustrianVineyards.com.

However, most applications are confronted with the criticism of being difficult to use: The needs of non-expert persons are not sufficiently met, their handling is often difficult, the visualisations hard to understand (Zeptner et al. 2021).

With ClimateCharts.net, a web app for climate geocommunication should be developed that is explicitly also appropriate for non-experts. With the application, points can be selected on a map for which interactive WalterLieth diagrams are shown. These should also be suitable for school lessons (Wiemann et al. 2016). Common rules of visualisation were considered for the presentation, and the diagrams are generated using the JavaScript library D3. However, temperature and precipitation are the only climate variables for which visualisations can currently be displayed (Zepner et al. 2021).

\section{Method}

The climate charts in AustrianVineyards.com are generated based on freely available data. These must first be obtained and processed so that they meet the requirements for the actual chart drawing, which is done analogous to ClimateCharts.net with the help of D3. Finally, the visualisations are integrated into the application AustrianVineyards.com.

\subsection{Data acquisition}

The data source for the climate data in AustrianVineyards.com is freely available data from the Central Institution for Meteorology and Geodynamics (ZAMG), which covers the whole territory of Austria, and the measurements are taken in a standardised way. In addition, the ZAMG updates the data annually, which ensures that the diagrams can be kept up to date in the future.

The ZAMG data are available as "yearbooks" in the CSV format and consist among others of the columns "Station," "Latitude," "Longitude," "Altitude," and "Parameter," by which for each station its position in the WGS 84 is known. However, the datasets of several climate stations cover different time periods. In addition, some stations were occasionally relocated, whereby the coordinates and elevation change, but their name usually remains the same. In this case, the change in position must be used to estimate whether stations with the same name and different coordinates can be treated as one. If this is the case, longer time series can be generated and the data basis for the calculation of derived variables such as mean values is more comprehensive.

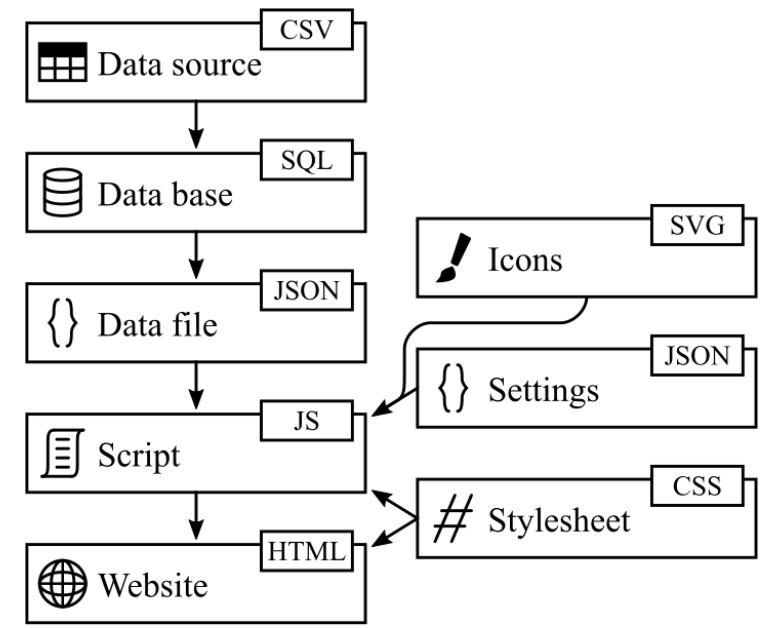

Figure 2. Schematic representation of the workflow from the data acquisition to the implementation of the climate visualisations in the AustrianVineyards.com website.

Such data cleaning steps are carried out when importing the ZAMG yearbooks into a database. Further calculations are carried out in this database, in which values are calculated for individual seasons, for example, as they are necessary for drawing the diagrams. The data required for individual climate diagrams are extracted from the database and stored in separate JSON files. They form the direct data basis for the diagram generation (Figure 2). 


\subsection{Data visualization}

The data is visualised using the JavaScript library DataDriven Documents, or D3 for short (D3.js), whereby an attempt is made to incorporate the current state of knowledge in the design of the diagrams. For each diagram type (parameter), there is a separate JavaScript file with an associated configuration file. The latter is read in by the script in addition to the actual data and is used to make diagram-specific settings. For example, colours, axis scaling and font sizes can be adjusted as required. This is intended to meet the demand for the highest possible flexibility, so that any meteorological measured variables can be visualised by high-quality climographs.

Currently, with line and bar charts two types of diagrams are used in AustrianVineyards.com. Basically, the line chart is primarily intended for interval-scaled data and the bar chart for ratio-scaled data. The icons that are part of the climate charts were created separately as vector files.

\subsection{Implementation in AustrianVineyards.com}

To be able to display the appropriate climate diagrams in AustrianVineyards.com for the corresponding wine regions of origin, it was necessary to assign the climate stations from which the climate data were taken to the wine-growing regions. For this purpose, the centroids of the origins were determined within a GIS and saved as a point layer. Then it was checked which climate stations were closest to which centroids of the origins, after which the allocation was made. Beforehand, it was ensured that only climate stations were considered which were relevant in the context of viticulture. For example, stations at too high an altitude or with insufficient data were removed.

\section{Results}

As a result, the climate data can be displayed in AustrianVineyards.com by selecting a wine origin on the map so that its info box opens. This box contains the climate data on temperature, precipitation, and sunshine duration in tabular form on a seasonal and full-year basis. In addition, the corresponding climate station is indicated. If the station name is clicked, it is displayed on the map with an icon as a point signature and the map centres on it (Figure 1). This clearly communicates the spatial reference on which the data is based.

The aspect of geo-communication is supplemented by climate communication by clicking on the respective parameter names in the info box. If that is done, a gallery with the climate diagrams opens, starting with the clicked parameter.

\subsection{Line chart}

Both positive and negative values can be displayed in the line diagram, as it is necessary for the temperature (Figure 3 ). Since the axis scaling is defined via the configuration file, it does not adapt to the values. In this way, diagrams with different spatial references should always remain visually comparable, even if their values differ widely from each other. While the measured values in the actual diagram area are shown on a monthly basis, in a season table in the lower right corner the data are shown on a seasonal basis. This is particularly significant regarding a wine vintage. In addition, the legend adapts to the values, according to which the entry identifying the reference year is ranked above or below the entry for the multi-year average, depending on whether above- or below-average values were observed in this year compared to the longterm observations.

Consequently, for the display of all temporal resolutions (monthly, seasonal, and annual basis), the values of the selected reference year can be directly compared with the average values measured over decades. To make it easier to recognise the higher values in the season table, the larger values are particularly highlighted by colour. Furthermore, specially designed icons for spring, summer, autumn, and winter are part of the season table. To ensure unambiguousness, they are labelled with their corresponding period (e.g., "Mar-May").

In the actual diagram area, in addition to the line of the reference year and a line to the long-term average, an area enclosed by the mean monthly extremes is also displayed. This is to illustrate which values lie within the range of possibility for the area associated with the climate station. In addition, the diagram heading is formed from the parameter and reference year. Below this are further information such as the name and geographical location of the weather station, both verbally and numerically by specifying the coordinates and altitude.

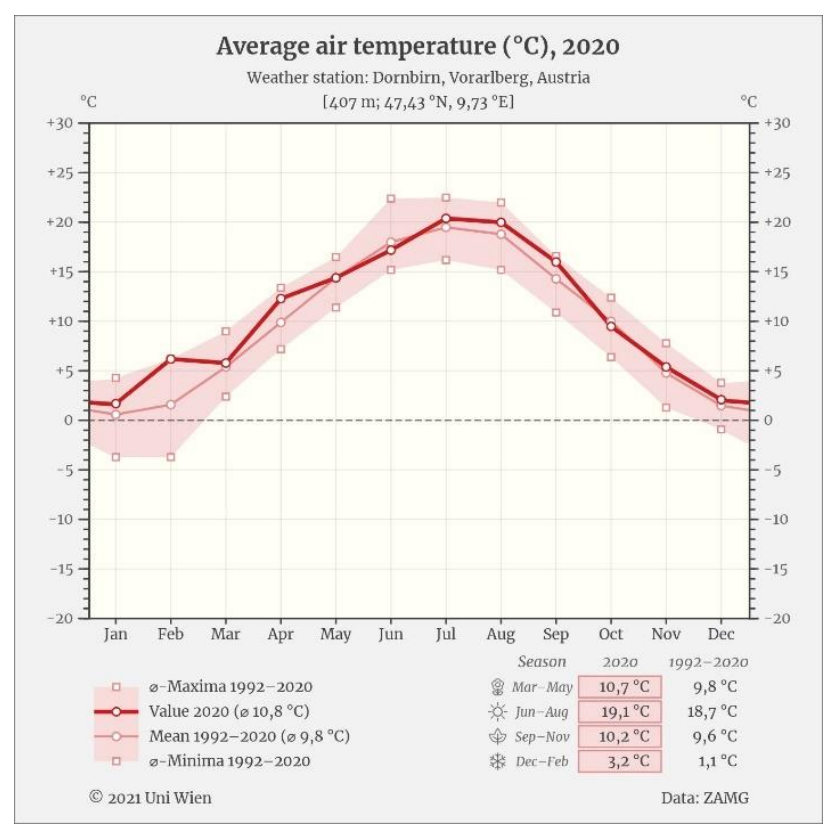

Figure 3. Line chart for communicating the temperature.

\subsection{Bar chart}

The basic structure of the bar charts corresponds to that of the line charts, i.e., the measurement data of a reference year as well as long-term data are communicated and visualised on a monthly, seasonal, and annual basis. Fixed axis scaling is also provided here so that diagrams of 
different locations can be directly compared visually with respect to the geo-communication.

In contrast to the line chart, the display of negative values is not provided, as the bar charts are designed for parameters for which sums are usually calculated (e.g., precipitation or sunshine duration). This, in conjunction with the y-axes being capped at the top, results in the problem of 'overshooting' values which is countered by labelling the upper ends of the overshooting bars with their numerical values and suggesting a bar break with a dashed line (Figure 4).

Due to a flexible structure, the diagrams can be adapted if needed, so that parameter-specific requirements can be met. For example, a darkened area was drawn in the chart for indicating the astronomical night (Figure 5). It is impossible for the sunshine duration bars to reach into this area. In that way, the diagram gains another level of information.

Although both the line and bar charts are designed to be interpretable without interaction, they can also be displayed interactively. In this case, the individual values are displayed when the mouse pointer is over a valueencoding mark. In addition, information on the copyright and the data source are placed in the lower corners of each chart. If someone hovers over them with the mouse, additional metadata is displayed.

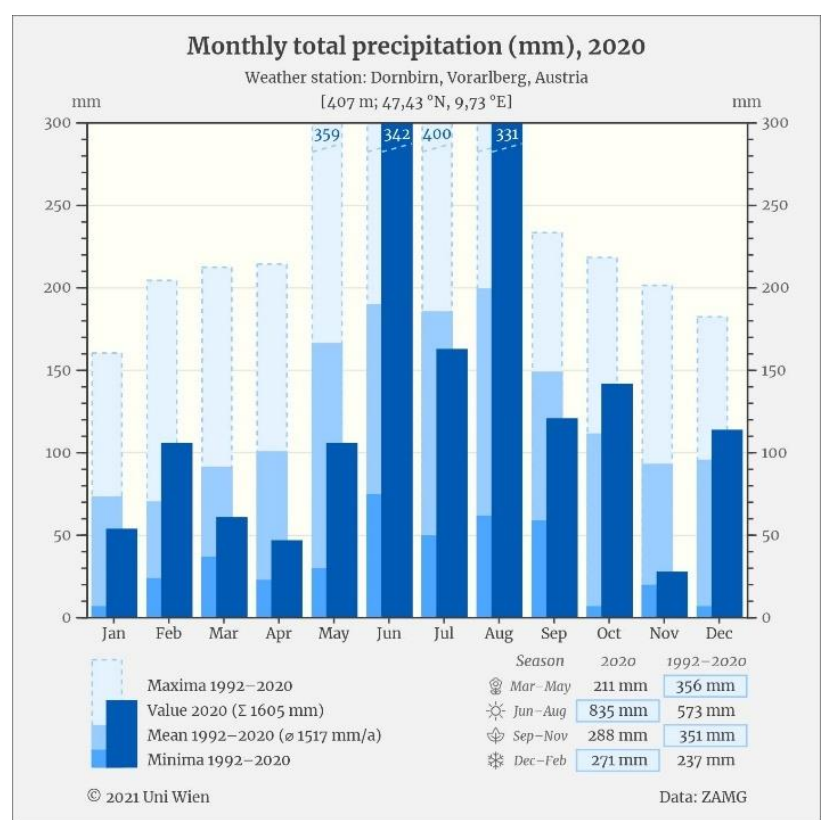

Figure 4. Bar chart for the communication of precipitation. Note the overshooting values

\section{Discussion}

The starting point was the question of a visualisation forms for communicating climate and weather data with respect to temporal as well as spatial aspects. Those should be able to display different meteorological parameters and be easy to read for a large audience of non-experts. Moreover, as a part of the marketing platform AustrianVineyards.com the graphs should correspond to an "image of excellence," which is why aesthetic aspects had to be considered.

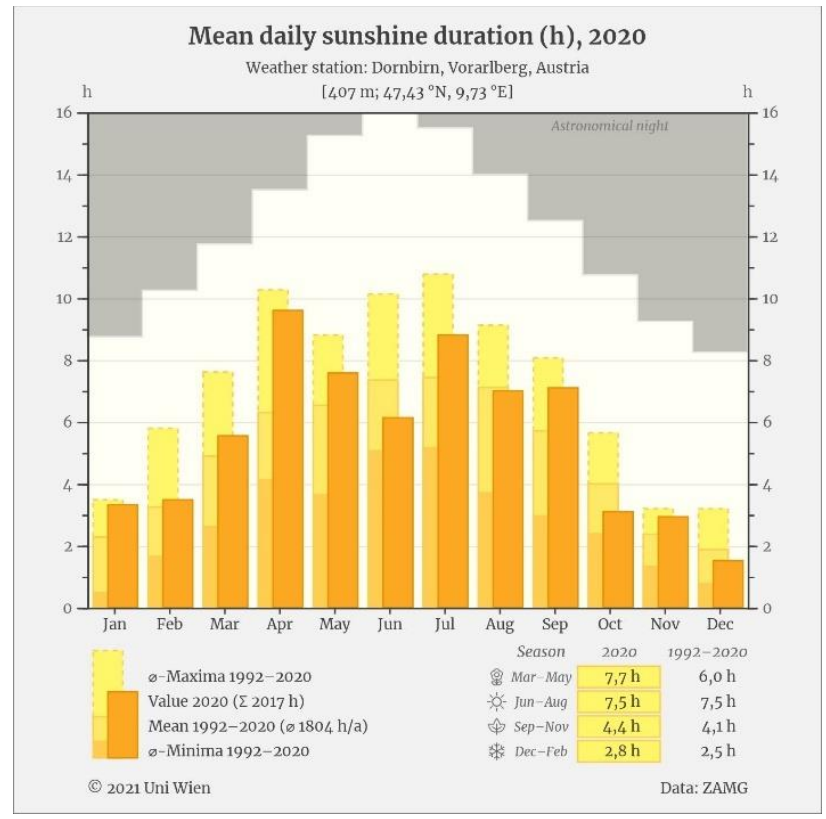

Figure 5. Bar chart to communicate the sunshine duration. Note the darkened area to indicate astronomical night.

The communication of the spatial reference was solved by assigning the weather stations to the respectively nearest wine-growing regions in AustrianVineyards.com. However, since in most cases the climate stations are located outside the wine origins, the possibility of displaying the exact location of the station in the map as a point signature was implemented. This takes up the concept of existing web-based climate communication applications of interactive display of climate stations on a map (OpenSensorWeb, Zeptner et al. 2021) but adapts it to the requirements of AustrianVineyards.com in that only the climate station that was assigned to the selected wine origin can be displayed.

As usual for climate charts, the diagrams designed in the course of this work also contain information on the location of the associated climate station. These are expressed both by the place name and the geographical coordinates. In this way, the location should also be apparent when the diagram is viewed without the map. In addition, the axes of the diagrams in AustrianVineyards.com are fixed scaled. This always ensures direct visual comparability and gives the implementation in AustrianVineyards.com a decisive feature about geo-communication in comparison to other comparable portals such as ClimateCharts.net or the Global Climate Monitor (Camarillo-Naranjo et al. 2019; Zeptner et al. 2021), where the diagram axes basically adapt to the values, which is why even diagrams with strongly diverging values can look similar.

The design principles outlined were taken into account in several ways: Important elements like the reference year are highlighted by darker colours. Larger values in the 
season table are visually emphasised. Interactive elements offer additional information without being necessary for interpretation. In addition, the specifically for the charts designed icons should help to quickly comprehend the season table. They are labelled to avoid misinterpretation. The parameter-specific colouring can be set (like other components) in the respective configuration file, so that they can be selected to promote an intuitive understanding of the graph.

In contrast to other climate communication platforms, the temporal aspect is given increased importance by always enabling the comparison of a reference year with the climate. In this way, even those who are not familiar with the local climate should be able to characterize a vintage.

For the future remains the possibility of including further parameters in AustrianVineyards.com, especially an extension would be possible through the modular diagram structure without extensive intervention in the code. In addition, the development of further diagram types as supplements to the line and bar chart should be considered to develop further visualisation proposals.

However, the integration of the interactive diagram versions in AustrianVineyards.com could be improved, especially since currently only static image files of the charts are displayed by default. For the interactive display, another click is necessary. This is because the chart handling does not work properly with touch input devices due to the small size of the interactive diagram components. An implementation of interactive diagrams which also allows a comfortable handling with small touchscreens like smartphones remains to be designed.

\section{References}

Allaby, M., 2019. climograph. In: A Dictionary of Plant Sciences, 4 ed, Oxford.

AustrianVineyards.com. https://austrianvineyards.com/, accessed on 14 August 2021.

Camarillo-Naranjo, J. M., Álvarez-Francoso, J. I., Limones-Rodríguez, Natalia; Pita-López, M. F. and Aguilar-Alba, M., 2019. The global climate monitor system: from climate data-handling to knowledge dissemination. In: International Journal of Digital Earth, vol. 12(4), pp. 394-414.

ClimateCharts.net. https://climatecharts.net/, accessed on 14 August 2021.

D3.js: Data-Driven Documents. https://d3js.org/, accessed on 11 August 2021.

Deutscher

Wetterdienst.

Klimadaten,

https://www.dwd.de/DE/service/lexikon/Functions/gloss ar.html?nn=103346\&lv2=101334\&lv3=649336,

accessed on 11 August 2021.

Earth Observation Monitor. http://earth-observationmonitor.net/, accessed on 11 August 2021.

Eichner, C., Nocke, T., Schulz, H.-J. and Schumann, H., 2015. Interactive Presentation of Geo-Spatial Climate Data in Multi-Display Environments. In: ISPRS Int. J. Geo-Inf, vol. 4(2), pp. 493-514.
Eberle, J., 2016. From global observations to local information: The Earth Observation Monitor. In: Free and Open Source Software for Geospatial (FOSS4G) Conference Proceedings, vol. 16, pp. 9-24.

Global Climate Monitor. https://www.globalclimatemonitor.org/, accessed on 14 August 2021.

Grant, R., 2019. Data Visualization. Charts, Maps, and Interactive Graphics, Boca Raton.

Harley, A., 2014. Icon Usability, https://www.nngroup.com/articles/icon-usability/, accessed on 11 August 2021.

Harold, J., Lorenzoni, I., Shipley, T. F. and Coventry, K. R., 2016. Cognitive and psychological science insights to improve climate change data visualization. In: Nature Climate Change, vol. 6, pp. 1080-1089.

Harper, J. and Agrawala, M., 2017. Converting Basic D3 Charts into Reusable Style Templates. In: IEEE Transactions on Visualization and Computer Graphics, vol. 24(3), pp. 1274-1286.

Kascak, L., Sanford, J. A., Braunstein, R. and Rebola, C., 2013. Icon Design to Improve Communication of Health Information to Older Adults. In: Communication Design Quarterly, vol. 2(1), pp. 6-32.

Kaye, N. R., Hartley, A. and Hemming, D., 2012. Mapping the climate: guidance on appropriate techniques to map climate variables and their uncertainty. In: Geoscientific Model Development, vol. 5, pp. 245-256.

Kim, D. H., Hoque, E. and Agrawala, M., 2020. Answering Questions about Charts and Generating Visual Explanations. In: Proceedings of the $2020 \mathrm{CHI}$ Conference on Human Factors in Computing Systems, Honolulu, HI, USA, pp. 1-13.

Kimball, M. A., 2013. Visual Design Principles: An Empirical Study of Design Lore. In: Journal of Technical Writing and Communication, vol. 43(1), pp. 3-41.

Kriz, K. and Pucher, A., 2021. AustrianVineyards.com the world's first nationwide information and presentation system of all wine estates. In: Proc. Int. Cartogr. Assoc., Florence, Italy.

Moody, D., 2009. The "Physics" of Notations: Toward a Scientific Basis for Constructing Visual Notations in Software Engineering. In: IEEE Transactions on Software Engineering, vol. 35(6), pp. 756-779.

Murray, S., 2017. Interactive Data Visualization for the Web. An Introduction to Designing with D3, Sebastopol.

Nocke, T., 2014. Images for Data Analysis: The Role of Visualization in Climate Research Processes. In: Schneider, B. and Nocke, T. (Ed.). Image Politics of Climate Change. Visualizations, Imaginations, Documentations, Bielefeld, pp. 55-78.

OpenSensorWeb.

https://www.opensensorweb.de/index.html, accessed on 14 August 2021.

Samsel, F., Wolfram, P., Bares, A., Turton, T. L. and Bujack, R., 2019. Colormapping resources and strategies 
for organized intuitive environmental visualization. In: Environmental Earth Sciences, vol. 78(9), pp. 1-12.

Schneider, B., 2012. Climate model simulation visualization from a visual studies perspective. In: WIREs Clim Change, vol. 3(2), pp. 185-193.

Stone, M. C., 2016. A Field Guide to Digital Color, Boca Raton.

Wiemann, F., Karrasch, P. and Müller, M., 2016. ClimateCharts.net - eine Webanwendung zur Erzeugung räumlich und zeitlich variabler Klimadiagramme. In: AGIT - Journal für Angewandte Geoinformatik, vol. 2, pp. 233-238.

Wrobel, M. and Reusser, D., 2014. Towards an Interactive Visual Understanding of Climate Change Findings on the Net: Promises and Challenges. In: Schneider, B. and Nocke, T. (Ed.). Image Politics of Climate Change. Visualizations, Imaginations, Documentations, Bielefeld pp. 187-210.

Zeileis, A. and Hornik, K., 2006. Choosing Color Palettes for Statistical Graphics. In: Research Report Series, vol. 41, Department of Statistics and Mathematics, Wirtschaftsuniversität Wien

Zeller, S. and Rogers, D. (2020): Visualizing Science: How Color Determines What We See. In: Eos, vol. 101.

Zeptner, L., Karrasch, P., Wiemann, F. and Bernard, L., 2021. ClimateCharts.net - an interactive climate analysis web platform. In: International Journal of Digital Earth, vol. 14(3). pp. 338-356. 